\title{
Pre-flare coronal dimmings ${ }^{\star}$
}

\author{
Q. M. Zhang ${ }^{1,2}$, Y. N. $\mathrm{Su}^{1}$, and H. S. Ji ${ }^{1}$ \\ 1 Key Laboratory for Dark Matter and Space Science, Purple Mountain Observatory, CAS, 210008 Nanjing, PR China \\ e-mail: zhangqm@pmo.ac.cn \\ 2 Key Laboratory of Solar Activity, National Astronomical Observatories, CAS, 100012 Beijing, PR China \\ Received 4 August 2016 / Accepted 28 November 2016
}

\begin{abstract}
Context. Coronal dimmings are regions of decreased extreme-ultravoilet (EUV) and/or X-ray (originally Skylab, then Yohkoh/SXT) intensities, which are often associated with flares and coronal mass ejections (CMEs). The large-scale impulsive dimmings have been thoroughly observed and investigated. The pre-flare dimmings before the flare impulsive phase, however, have rarely been studied in detail.

Aims. We focus on the pre-flare coronal dimmings. We report our multiwavelength observations of the GOES X1.6 solar flare and the accompanying halo CME that was produced by the eruption of a sigmoidal magnetic flux rope (MFR) in NOAA active region (AR) 12158 on 2014 September 10.

Methods. The eruption was observed by the Atmospheric Imaging Assembly (AIA) on board the Solar Dynamic Observatory (SDO) The photospheric line-of-sight magnetograms were observed by the Helioseismic and Magnetic Imager (HMI) on board SDO. The soft X-ray (SXR) fluxes were recorded by the GOES spacecraft. The halo CME was observed by the white-light coronagraphs of the Large Angle Spectroscopic Coronagraph (LASCO) on board SOHO.

Results. About $96 \mathrm{~min}$ before the onset of the flare/CME, narrow pre-flare coronal dimmings appeared at the two ends of the twisted MFR. They extended very slowly, with their intensities decreasing with time, while their apparent widths (8-9 Mm) continued to be nearly constant. During the impulsive and decay phases of flare, typical fan-like twin dimmings appeared and expanded, with a much larger extent and lower intensities than the pre-flare dimmings. The percentage of the $171 \AA$ intensity decrease reaches $40 \%$. The pre-flare dimmings are most striking in 171,193 , and $211 \AA$ with formation temperatures of $0.6-2.5$ MK. The northern part of the pre-flare dimmings could also be recognized in 131 and $335 \AA$.

Conclusions. To our knowledge, this is the first detailed study of pre-flare coronal dimmings; they can be explained by density depletion as a result of the gradual expansion of the coronal loop system surrounding the MFR during the slow rise of the MFR.
\end{abstract}

Key words. Sun: corona - Sun: coronal mass ejections (CMEs) - Sun: flares

\section{Introduction}

Flares and coronal mass ejections (CMEs) are the most violent processes of energy release in the solar atmosphere and the most important sources of space weather (Forbes et al. 2006; Chen 2011; Hudson 2011; Fletcher et al. 2011). Sometimes, the preflare phase, rise phase, and decay phase of flares are temporally related to the initial phase, impulsive acceleration phase, and propagation phase of CMEs, respectively (Zhang et al. 2001). Flares and CMEs are often associated with the eruptions of filaments (Zhang et al. 2015, 2016), which are suspended by magnetically sheared arcades or magnetic flux ropes (MFRs) along the polarity inversion lines (Titov \& Démoulin 1999; Aulanier et al. 2010; Xia et al. 2014; Su et al. 2015). An MFR consists of a set of magnetic field lines that is wrapped around the central axis of the rope and appears as a hot channel with temperatures as high as $10 \mathrm{MK}$ in the low corona (Zhang et al. 2012a). The large-scale eruptions typically create global disturbances that propagate across very long distances on the solar surface. For the first time, Thompson et al. (1998) observed dimming regions and bright wavefronts with enhanced intensities that propagate quasi-radially from the source region of a CME. The onset of dimming is consistent with the initiation of

\footnotetext{
* Movie associated to Fig. 5 is available at http: //www . aanda.org
}

the wavefront, that is, the coronal wave or extreme-ultraviolet (EUV) wave (Chen et al. 2002; Patsourakos \& Vourlidas 2012). The decrease in intensity of the dimming region is due to a decrease in plasma density rather than in temperature (Harrison \& Lyons 2000). The percentage of the density decrease could reach $35 \%-40 \%$ (Jin et al. 2009). The temperatures of the EUV dimmings are 1-4 MK (Zhukov \& Auchère 2004; Robbrecht \& Wang 2010; Cheng et al. 2012a). Thompson et al. (2000) identified transient dimming regions with strong EUV emission depletion around the eruption, and the dimming areas are cospatial with the footprints of CMEs (Bewsher et al. 2008; Attrill et al. 2010). As the CME proceeds, the EUV wave propagates outward at a speed of $50-1500 \mathrm{~km} \mathrm{~s}^{-1}$ and the area of trailing dimming increases, covering a significant fraction of the solar surface (Harra et al. 2007; Thompson \& Myers 2009; Attrill \& Wills-Davey 2010; Cheng et al. 2012b). Recent quadrature observations have revealed the dome-shaped nature of EUV waves and dimming regions (Patsourakos et al. 2009; Veronig et al. 2010). There is a special type of dimmings that appear near the two ends of a pre-flare, S-shaped sigmoid at the beginning of the impulsive acceleration phase of CMEs (Miklenic et al. 2011). Such twin dimmings, following the eruption of a twisted MFR, can also persist for tens of hours to more than three days (Sterling \& Hudson 1997; Kahler \& Hudson 2001). The decrease of the twin dimming intensity is correlated 


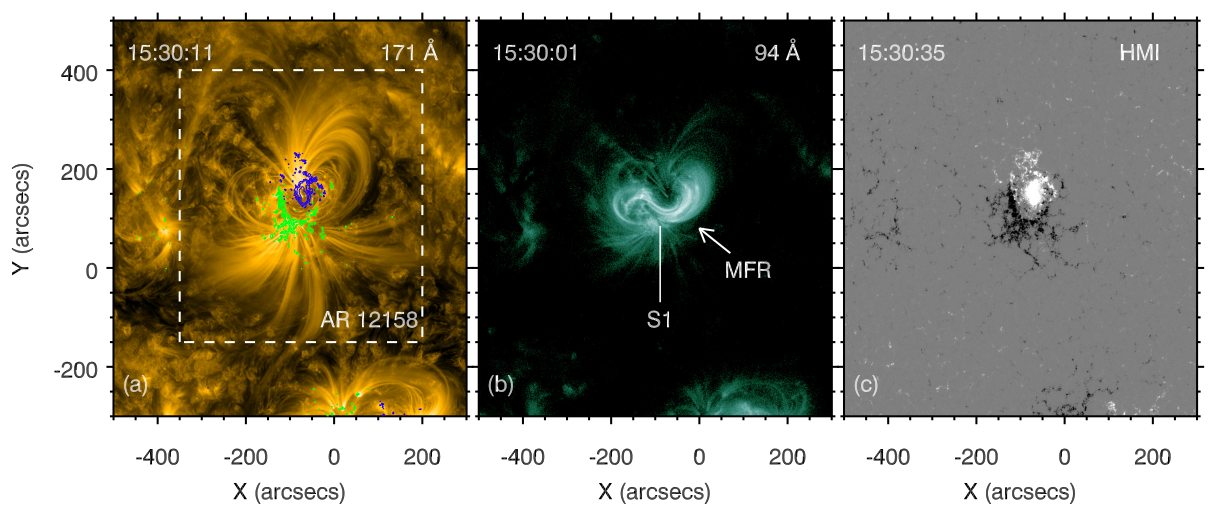

Fig. 1. a) AIA $171 \AA$ image at $15: 30: 11$ UT. The AR 12158 is enclosed by the white dashed box. b) AIA $94 \AA$ image at 15:30:01 UT, which features an MFR pointed out by the white arrow. A vertical slice $\mathrm{S} 1$ (white solid line) is used to investigate the slow rise and expansion of the coronal loops. c) HMI LOS magnetogram at 15:30:35 UT. The contours $( \pm 400, \pm 800$, and $\pm 1200 \mathrm{G})$ of the LOS positive and negative magnetic field are overlaid on the $171 \AA$ image with blue and green lines, respectively. with the increase in flaring arcade intensity in EUV wavelengths (Zarro et al. 1999). Interestingly, twin dimming regions are found to rotate around the center of the flare site as a result of the rotation of the erupting filament (Miklenic et al. 2011). Similar to typical coronal dimmings that have a circular or elliptical shape, twin dimmings also originate from density depletion as a result of magnetic field line stretching or reconfiguration associated with CMEs. Spectroscopic observations from space telescopes have revealed strong plasma upflows at speeds of tens to hundreds of $\mathrm{km} \mathrm{s}^{-1}$ in the coronal dimming regions (e.g., Harra \& Sterling 2001; Dolla \& Zhukov 2011). The gradual recovery of dimming regions lasts for a long time by reestablishment of the bright coronal loops (Reinard \& Biesecker 2008; Attrill et al. 2010). Sophisticated numerical simulations have also improved our understandings of coronal waves and dimmings. Imada et al. (2007) discovered that the upflow velocities $\left(16-160 \mathrm{~km} \mathrm{~s}^{-1}\right)$ in the dimming region depend on the formation temperatures of the emission lines, that is, hotter lines show faster upflow velocities. Imada et al. (2011) found that the temperature-dependent upflow in the dimming region can be well modeled by quasi-steady flow in a vertical flux tube whose cross section expands super-radially with height from the solar surface.

Since the launch of Solar Dynamic Observatory (SDO), coronal dimmings following CMEs have been extensively investigated (Liu \& Ofman 2014; Warmuth 2015, and references therein). However, pre-flare or pre-eruption dimmings have rarely been reported. Cheng \& Qiu (2016) studied the coronal dimmings on 2011 December 26 in detail and found that the rapid dimmings started after the onset of fast magnetic reconnection and CME acceleration. The authors concluded that at some locations, the gradual dimmming started about $30 \mathrm{~min}$ before the CME eruption and impulsive flare reconnection. They proposed that the gradual and weak pre-eruption dimming may reflect the slow expansion of coronal structures. In this paper, we report long-term EUV dimmings before the onset of flare and CME observed by the Atmospheric Imaging Assembly (AIA; Lemen et al. 2012) aboard SDO. On 2014 September 10 , an inverse-S, sigmoidal MFR formed in NOAA active region (AR) 12158 close to the disk center. At $\sim 17: 21 \mathrm{UT}$, the MFR erupted and produced a GOES X1.6 flare that peaked at $\sim 17: 45$ UT and a full-halo CME that propagated toward Earth (Li et al. 2015a,b; Li \& Zhang 2015; Cheng et al. 2015; Zhao et al. 2016; Dudík et al. 2016). This paper is structured as follows. The data analysis is described in Sect. 2, and the results are shown in Sect. 3. We discuss the nature and significance of pre-flare dimmings in Sect. 4. Finally, we summize the results in Sect. 5 .

\section{Data analysis}

The eruption was clearly observed by AIA, which has seven EUV filters $(94,131,171,193,211,304$, and $335 \AA$ ) with a cadence of $12 \mathrm{~s}$ and two UV filters (1600 and $1700 \AA$ ) with a cadence of $24 \mathrm{~s}$. Line-of-sight (LOS) magnetograms from the Helioseismic and Magnetic Imager (HMI; Scherrer et al. 2012) on board SDO with a cadence of $45 \mathrm{~s}$ were used to study the photospheric magnetic field of the AR. The full-disk EUV images and magnetograms have spatial resolutions of 1'!2 and $1^{\prime \prime}$, respectively. The AIA and HMI level_1 fits data were calibrated using the standard Solar Software (SSW) programs aia_prep.pro and hmi_prep.pro. The fast halo $\mathrm{CME}^{1}$ at a linear speed of $1267 \mathrm{~km} \mathrm{~s}^{-1}$ was observed by the $\mathrm{C} 2$ and $\mathrm{C} 3$ white-light (WL) coronagraphs of the Large Angle Spectroscopic Coronagraph (LASCO; Brueckner et al. 1995) on board SOHO. The $\mathrm{LASCO} / \mathrm{C} 2$ images were calibrated using the $S S W$ program c2_calibrate.pro. The high-cadence (2.047 s) SXR fluxes of the flare were recorded by GOES.

\section{Results}

Panel a in Fig. 1 shows the $171 \AA$ image at 15:30:11 UT before the onset of flare. The AR 12158 is enclosed by the white dashed box $\left(550^{\prime \prime} \times 550^{\prime \prime}\right)$. Panel b shows the $94 \AA$ image at 15:30:01 UT with a much higher formation temperature $(\sim 6 \mathrm{MK})$. The image features the bright inverse-S sigmoidal MFR embedded in the core of AR (Cheng et al. 2015). The photospheric LOS magnetogram observed by HMI at 15:30:35 UT is displayed in panel c. The AR was associated with a complex $\beta \gamma \delta$ sunspot. The contours $( \pm 400, \pm 800$, and $\pm 1200 \mathrm{G})$ of the photospheric LOS magnetic field of the positive and negative polarities are overlaid on the $171 \AA$ image with blue and green lines, respectively. It is clear that the MFR resides between the positive and negative polarities.

In Fig. 2 the GOES SXR light curves in 0.5-4.0 $\AA$ and 1-8 $\AA$ are plotted in panel a with dashed and solid lines. The SXR fluxes increase gradually from $\sim 15: 45$ UT and rise rapidly during the impulsive phase of the flare before decreasing slowly during the long decay phase ( $\mathrm{Li}$ et al. 2015a). We also calculated the integrated base-difference EUV intensities of the whole AR within the white box of Fig. 1a. The temporal evolutions of the normalized intensities during 15:30-18:30 UT are depicted with colored lines in Fig. 2b. It is clear that the total EUV intensities of the AR increase very slowly during the pre-flare phase

\footnotetext{
1 http://cdaw.gsfc.nasa.gov/CME_list/UNIVERSAL/2014_ 09/
} 


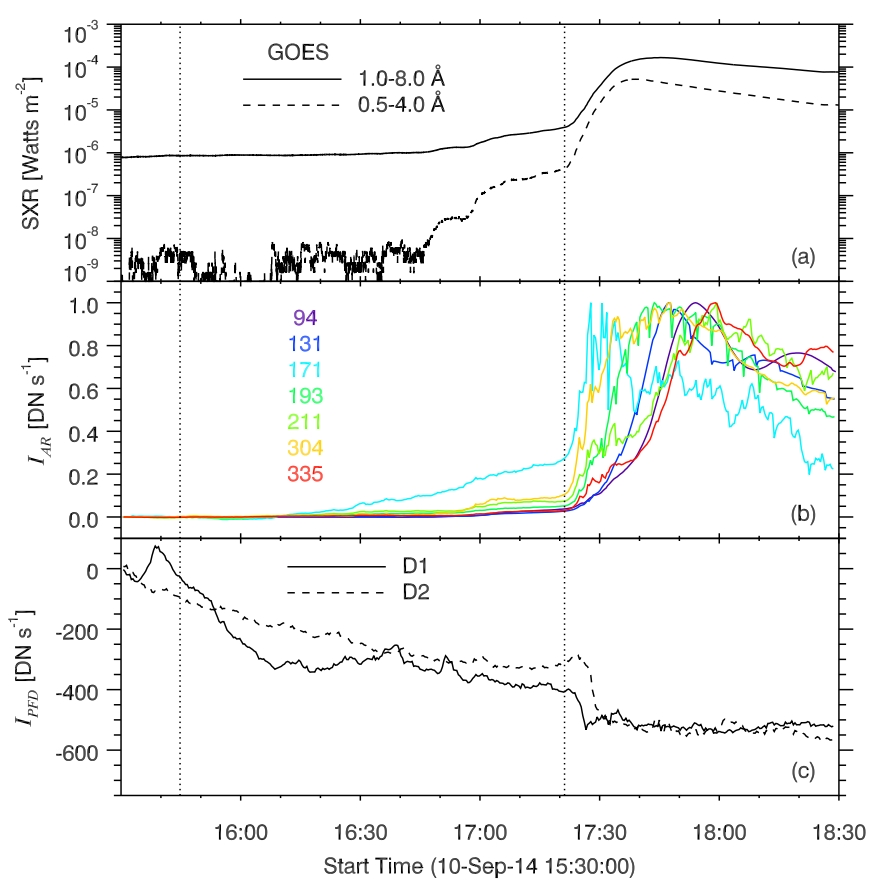

Fig. 2. a) GOES SXR fluxes of the flare during 15:30-18:30 UT. b) Temporal evolutions of the normalized, integral intensities of the AR within the dashed box of Fig. 1a. c) Temporal evolutions of the $171 \AA$ base-difference intensities of D1 and D2 marked in Fig. 5b. PFD stands for pre-flare dimming. The two vertical dotted lines in each panel denote the start time (15:45 UT) and end time (17:21 UT) of pre-flare dimmings.

(15:45-17:21 UT), which is similar to the SXR light curves. The slow rises of both SXR and EUV fluxes of the AR suggest that plasma heating as a result of the release of magnetic free energy is already taking place, presumably through magnetic reconnection (Cheng et al. 2015). The early heating is also supported by the fact that the 94 and $131 \AA$ light curves with higher peak formation temperatures do not start to increase until later.

In Fig. 1b we draw a vertical slice (S1), which originates from the flare site with a length of $112 \mathrm{Mm}$. The temporal evolutions of the intensities along S1 in four of the AIA filters during 15:30-18:30 UT are displayed as time-slice diagrams in Fig. 3. It is clear that the coronal loops are undergoing slow rise and expansion during the mild heating of the AR before the onset of the flare, especially in 193 and $211 \AA$.

The halo CME appeared and expanded isotropically in the field of view of LASCO/C2 during 18:00-18:36 UT. In Fig. 4 the base-difference WL image of the CME at $18: 12$ UT is displayed in the left panel. The height-time profile of the CME is plotted in the right panel with diamonds. The result of a leastsquares fitting of the profile is overlaid with a dashed line. The extrapolated initiation time of CME coincided with the onset of flare at $\sim 17: 21 \mathrm{UT}$.

Taking the EUV images at $\sim 15: 30$ UT as base images, we obtained the subsequent base-difference images until 18:30 UT (see the online movie). Panels a-e in Fig. 5 show five snapshots of the base-difference images in $171 \AA$. It is clear that narrow dimming regions appeared at the northern and southern ends of the MFR before flare, which are denoted by "pre-flare dimming" in panels $a$ and $b$. The pre-flare dimmings eventually extended, especially for the northern part in panel b. During the impulsive phase of flare when the MFR erupted out of the corona,

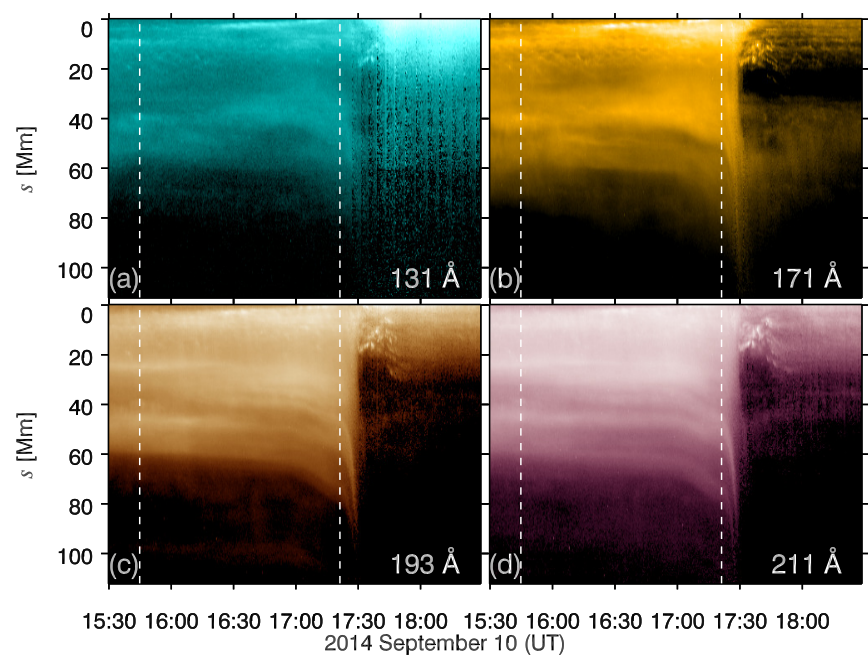

Fig. 3. Time-slice diagrams of $\mathrm{S} 1$ in four AIA filters using the original images. $s=0 \mathrm{Mm}$ represents the flare site. The two vertical dashed lines in each panel signify the start time (15:45 UT) and end time (17:21 UT) of pre-flare dimmings.
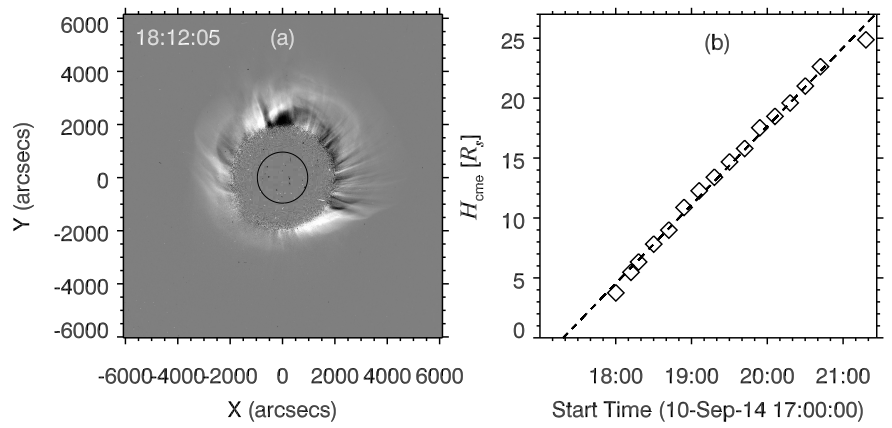

Fig. 4. a) WL base-difference image observed by $\mathrm{LASCO} / \mathrm{C} 2$ at 18:12:05 UT. b) Height-time profile of the halo CME. The dashed line represents the least-squares linear fitting of the profile.

two bright ribbons were detected in the $171 \AA$ and $1600 \AA$ images (see panels $c$ and $\mathrm{f}$ ). Meanwhile, the areas of the dimming regions expanded rapidly while their intensities decreased to a great extent, forming the typical fan-like "twin dimming" indicated in panels $d$ and e. The lack of bright loops nearby in the base-difference images suggests that this is most likely a density depletion. During the decay phase of flare, the dimmings were sustained and extended very slowly. In panel e, the $94 \AA$ intensity contours are superposed on the $171 \AA$ image with red lines. It is evident that both the pre-flare dimmings and twin dimmings originated at the two ends of the sigmoid. This is consistent with the finding that the observed coronal dimmings are located around the footpoints of a erupting flux rope (Su et al. 2011).

To illustrate the temporal evolution of the dimmings more clearly, we selected a curved slice (S0 with dashed green line) that passes through the core of $\mathrm{AR}$ and dimming regions in Fig. 5e. The total length of S0 is $327.5 \mathrm{Mm}$. The evolutions of the base-difference intensities in 171, 193, and $211 \AA$ along S0 are displayed as time-slice diagrams in the top panels of Fig. 6 . The pre-flare dimmings and twin dimmings are both distinct as dark regions in the diagrams, especially in $171 \AA$, indicating that the plasma dominantly involved in the dimmings was in the range of 0.6-2.5 MK. The pre-flare dimmings started at $\sim 15: 45 \mathrm{UT}$ 

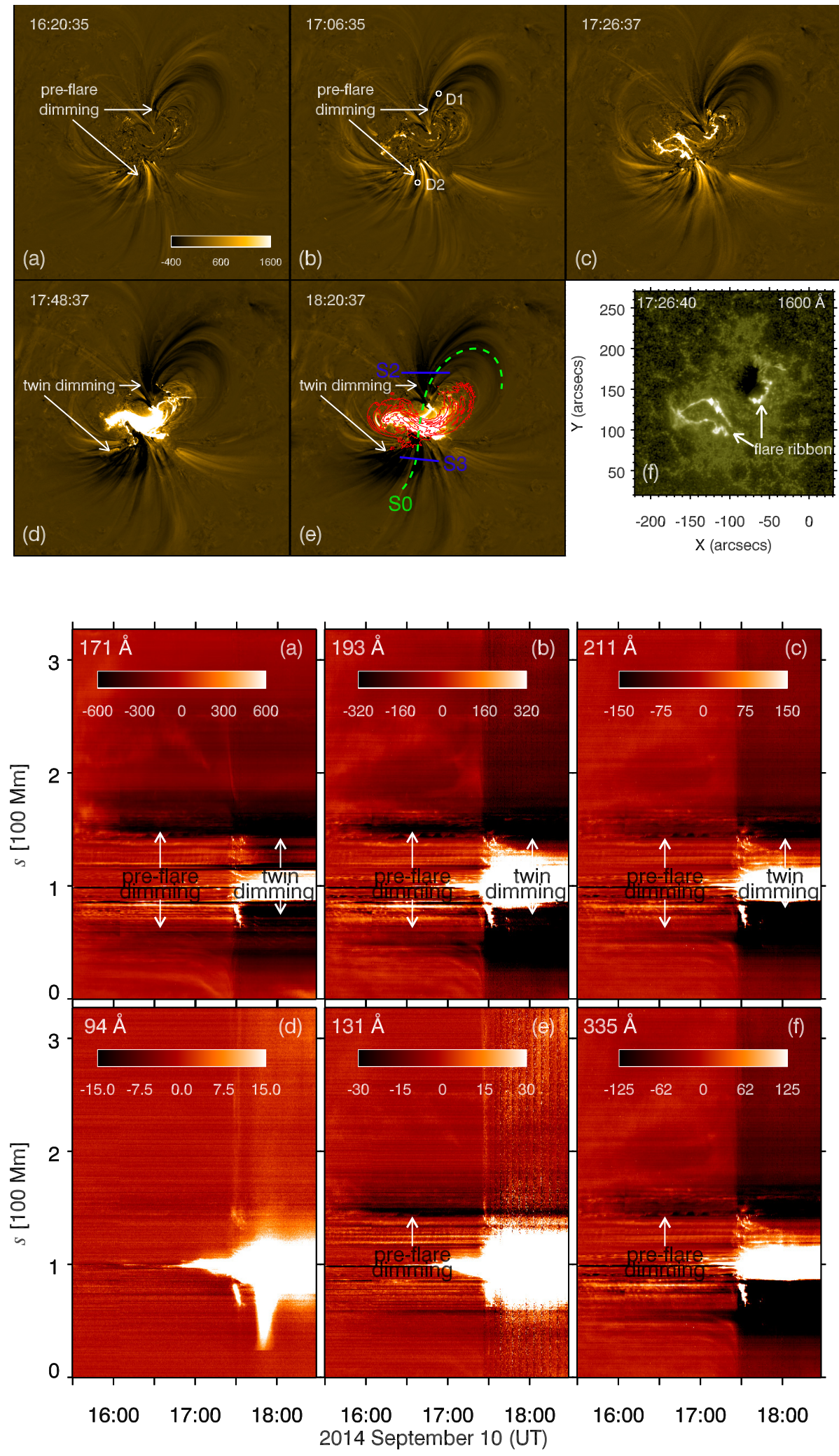

Fig. 5. a)-e) Snapshots of the base-difference images in $171 \AA$. The pre-flare dimmings and twin dimmings are pointed out by the white arrows. In panel b), two circles mark the locations of two representative pre-flare dimmings (D1 and D2). In panel e), a curved slice S0 (green dashed line) is used to investigate the temporal evolution of the coronal dimmings. The intensity contours of the $94 \AA$ image in Fig. $1 \mathrm{~b}$ are overlaid with red lines. f) AIA $1600 \AA$ image at 17:26:40 UT that features two bright flare ribbons. The temporal evolution of the flare is shown in a movie available online.

Fig. 6. Time-slice diagrams of $\mathrm{S} 0$ in 171, 193, $211,94,131$, and $335 \AA$ using the AIA basedifference images. $s=0$ and $s=327.5 \mathrm{Mm}$ represent the southeastern and northwestern endpoints of S0. The dark pre-flare dimmings and twin dimmings are pointed out by the white arrows in each panel.

and lasted for $\sim 96$ min until the beginning of the flare impulsive phase at $\sim 17: 21$ UT. Afterward, twin dimmings were observed and expanded rapidly with much larger extent and lower intensities. The percentage of intensity decrease is $\sim 40 \%$. The northern part of the pre-flare dimmings was always more striking and evident than the southern part, while the southern part of the twin dimmings was always stronger than the northern part during the flare. The evolutions of the base-difference intensities in 94, 131, and $335 \AA$ along S0 are displayed as time-slice diagrams in the bottom panels of Fig. 6. Only the northern part of the pre-flare dimmings could be identified with slightly lower magnitudes than those in the cooler filters, while the southern part is hardly visible in these wavelengths.

To compare the pre-flare dimming regions with the whole AR, we selected two representative positions (D1 and D2) in the pre-flare dimming regions, which are labeled with small circles 


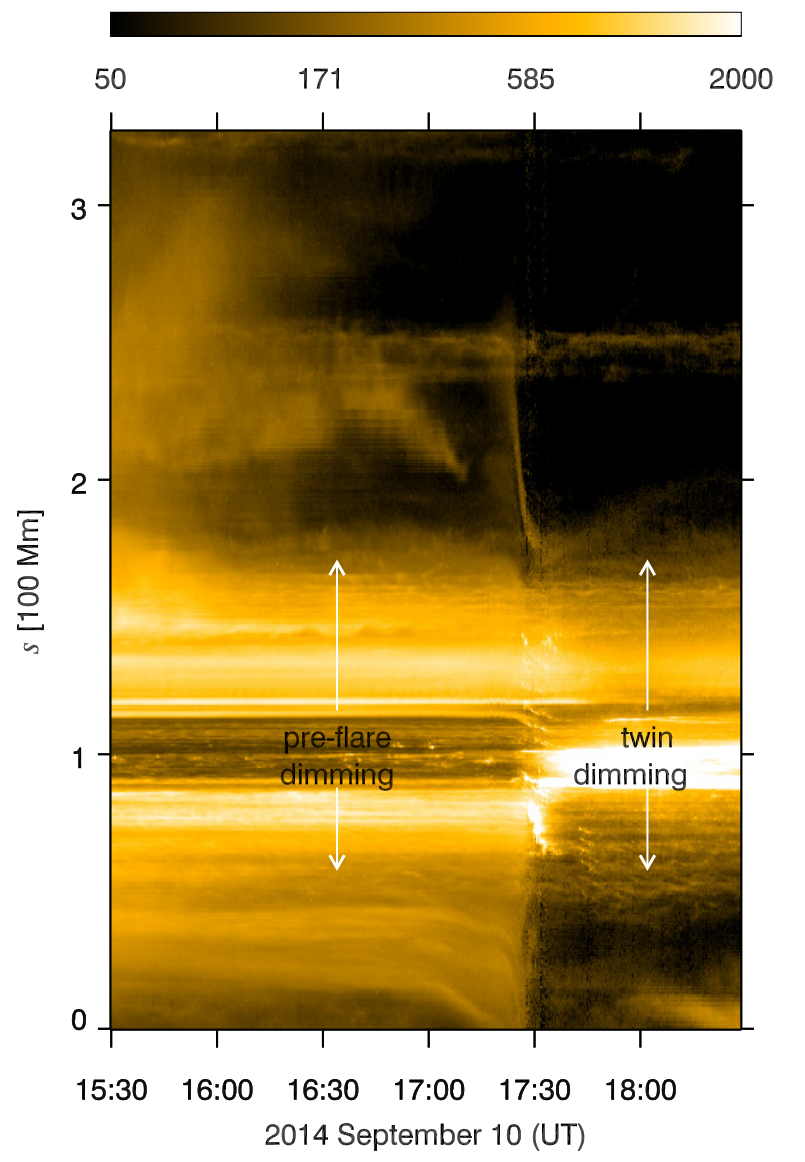

Fig. 7. Time-slice diagram of S0 derived from the original $171 \AA$ Amages instead of the base-difference images. $s=0 \mathrm{Mm}$ represents the southeastern endpoint of S0. The dark pre-flare dimmings and twin dimmings are pointed out by the arrows.

in Fig. 5b. The temporal evolutions of the base-difference intensities of D1 and D2 in $171 \AA$ are displayed in Fig. 2c with solid and dashed lines, respectively. It is obvious that the intensities of D1 and D2 gradually decrease from $\sim 15: 45$ UT until 17:27 and 17:30 UT, respectively.

\section{Discussion}

\subsection{Nature of pre-flare dimmings}

Despite extensive observations and investigations in the past, the origins of coronal dimmings are not completely understood. Mason et al. (2014) summarized the mechanisms of coronal dimmings, including the mass-loss dimmings as a result of the ejection of emitted plasma with temperatures of several MK (Harrison \& Lyons 2000), thermal dimmings because different EUV filters have different formation temperatures (Cheng et al. 2011), and Doppler dimming, to only name a few. In our study, the large-scale twin dimmings followed the pre-flare dimmings. It is widely accepted that the twin dimmings originate from the density depletion that is a result of eruption of an MFR (Su et al. 2011). Before the flare impulsive phase, there was no clear signature of mass loss. The pre-flare dimmings result from density depletion when the whole coronal loop system surrounding the MFR undergoes gradual volume expansion during the slow rise of the MFR. Such a precursor expansion in this event has also been noticed by Dudík et al. (2016). As illustrated in the schematic cartoon of Cheng et al. (2015), the
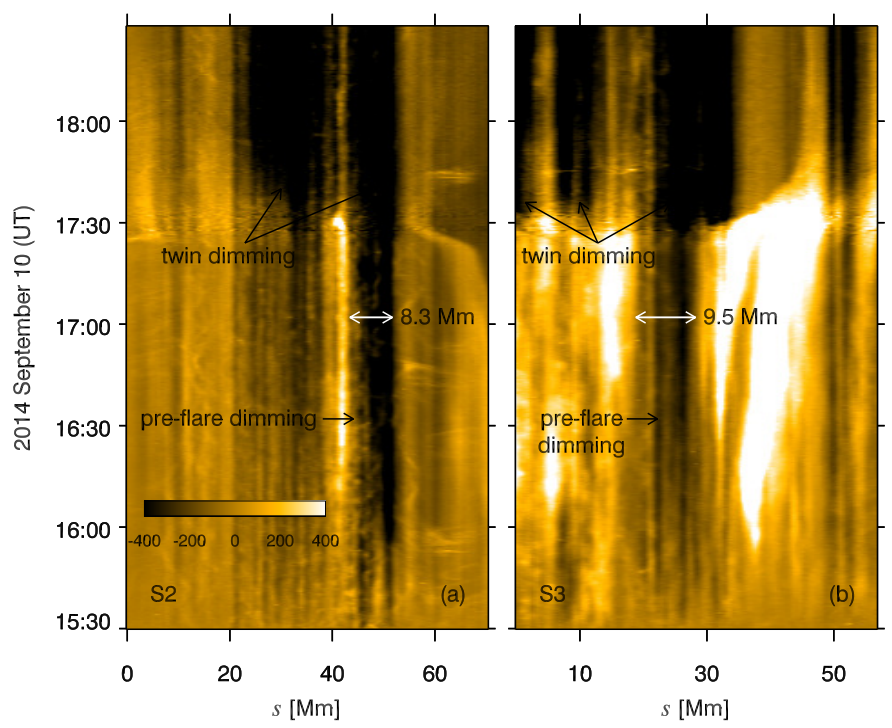

Fig. 8. Time-slice diagrams of S2 (left panel) and S3 (right panel) in $171 \AA$ using the AIA base-difference images. $s=0 \mathrm{Mm}$ represents the left endpoints of S2 and S3. The dark pre-flare dimmings and twin dimmings are pointed out by the black arrows in each panel. The white double-headed arrows denote the apparent widths of the pre-flare dimmings along S2 and S3.

tether-cutting magnetic reconnection occurred during the preflare phase (Moore et al. 2001), which has two effects. One is the mild heating of the MFR residing in the core of AR (see Fig. 2a). The other is the decreased constraint on the MFR from the envelop magnetic field and the increase in poloidal magnetic flux of the MFR at the same time, leading to the slow rise of the MFR (Sterling \& Moore 2005; Chifor et al. 2007; Sterling et al. 2011). This is usually accompanied by gradual volume expansion since the magnetic field and electron number density decrease with height in the low- $\beta$ corona.

Considering that both pre-flare dimmings and twin dimmings are clearly visible in the base-difference images, there is a concern that the pre-flare dimmings might be artifacts of the base-difference image processing. To dispel this doubt, we made a time-slice diagram of S0 using the original images in $171 \AA$ instead of the base-difference images. It is obvious from Fig. 7 that pre-flare dimmings and twin dimmings are both distinguishable as dark regions. The locations and times of the dimmings are the same as those in Fig. 6a. Therefore, the pre-dimmings are real and convincing, not an artifact of the base-difference image processing.

Another concern is that the pre-flare dimmings might result from the transverse shifts of the bright adjacent coronal loops before its impulsive eruption of the MFR. To test this conjecture, we selected two short straight slices (S2 and S3 with solid blue lines) that cross the northern and southern parts of the dimming regions in Fig. 5e. The total lengths of S2 and S3 are 70.6 and 57.3 Mm. The evolutions of the base-difference intensities in $171 \AA$ along S2 and S3 are displayed as time-slice diagrams in the left and right panels of Fig. 8. We note that the time axis and distance axis are different from those of Fig. 6. Starting from $\sim 15: 45$ UT, the northern part of pre-flare dimmings decreases in intensity while its apparent width $(\sim 8.3 \mathrm{Mm})$ is nearly kept constant. After the onset of flare, the northern part of twin dimmings expanded rapidly to a much broader extent with lower intensity (see panel a). Similar to the northern part, the southern part of pre-flare dimmings decreases in intensity while the 
apparent width $(\sim 9.5 \mathrm{Mm})$ is kept constant before the southern part of the twin dimmings appears (see panel b). There is no clear signature of coronal loop shifts in the transverse directions before the impulsive phase of flare.

\subsection{Significance of pre-flare dimmings}

Although it is difficult to predict when a filament (flux rope) erupts that might lead to a flare and/or a CME, there are precursor activities a few to tens of minutes before the eruptions, such as initial heating and brightenings as a result of magnetic reconnection (Sterling \& Moore 2005; Joshi et al. 2011), a strong blue asymmetry in the $\mathrm{H} \alpha$ line (Cho et al. 2016), a radio noise storm that is due to reconstruction of the large-scale magnetic structure (Lantos et al. 1981), and filament oscillation (Zhang et al. $2012 \mathrm{~b}$ ), to only name a few. In this paper, we observed longterm ( $\sim 96 \mathrm{~min})$ coronal dimmings before the onset of the impulsive phase of the flare and the initiation of the halo CME, which can be considered as another precursor of solar eruptions. Additional case studies and statistical works using multiwavelength observations are worthwhile to investigate the nature of pre-flare coronal dimmings. If the proposal is consolidated, it will be undoubtedly valuable for space weather prediction.

\section{Summary}

We have reported our multiwavelength observations of the flare/CME event that occurred in AR 12158 as a result of an erupting MFR on 2014 September 10, focusing on the study of pre-flare coronal dimmings. The pre-flare dimmings appeared at the two ends of the twisted MFR and lasted for $\sim 96$ min until the onset of the flare and initiation of the halo CME. The narrow pre-flare dimmings extended very slowly while their intensities decreased with time. Their apparent widths $(8-9 \mathrm{Mm})$ did not change strongly, however. The pre-flare dimmings are observed by SDO/AIA mainly in 171,193 , and $211 \AA$ with formation temperatures of $0.6-2.5 \mathrm{MK}$. The northern part of preflare dimmings could be identified in 131 and $335 \AA$ with higher formation temperatures. During the impulsive and decay phases of the flare, typical fan-like twin dimmings appeared and expanded with much larger extent and lower intensities than the pre-flare dimmings. The percentage of the $171 \AA$ intensity decrease reaches $\sim 40 \%$. It is likely that the pre-flare dimmings are progenitors of the large-scale twin dimmings. We conclude that the pre-flare dimmings originate from density depletion that is a result of the gradual expansion of the coronal loop system surrounding the MFR during the slow rise of the MFR. Our findings provide strong support for the conjecture of Cheng \& Qiu (2016) and have potential significance for space weather prediction.

Acknowledgements. The authors sincerely thank the referee for detailed and valuable comments. We also thank J. Zhang, M. D. Ding, P. F. Chen, R. Liu, B. Kliem, G. Aulanier, and X. Cheng for fruitful discussions. SDO is a mission of NASA's Living With a Star Program. AIA and HMI data are courtesy of the NASA/SDO science teams. SOHO is a project of international cooperation between ESA and NASA. Q.M.Z. is supported by the Surface Project of Jiangsu No. BK20161618, NSFC No. 11303101, 11333009, 11573072, and the open research program of Key Laboratory of Solar Activity, National Astronomical Observatories, CAS No. KLSA201510. H.S.J. is supported by the Strategic Priority Research Program-The Emergence of Cosmological Structures of the CAS, Grant No. XDB09000000. Y.N.S. is supported by NSFC 11473071, Youth Fund of Jiangsu BK20141043, and the One Hundred Talent Program of Chinese Academy of Sciences.

\section{References}

Attrill, G. D. R., \& Wills-Davey, M. J. 2010, Sol. Phys., 262, 461 Attrill, G. D. R., Harra, L. K., van Driel-Gesztelyi, L., \& Wills-Davey, M. J. 2010, Sol. Phys., 264, 119

Aulanier, G., Török, T., Démoulin, P., \& DeLuca, E. E. 2010, ApJ, 708, 314

Bewsher, D., Harrison, R. A., \& Brown, D. S. 2008, A\&A, 478, 897

Brueckner, G. E., Howard, R. A., Koomen, M. J., et al. 1995, Sol. Phys., 162, 357

Chen, P. F. 2011, Liv. Rev. Sol. Phys., 8, 1

Chen, P. F., Wu, S. T., Shibata, K., \& Fang, C. 2002, ApJ, 572, L99

Cheng, J. X., \& Qiu, J. 2016, ApJ, 825, 37

Cheng, X., Zhang, J., Liu, Y., \& Ding, M. D. 2011, ApJ, 732, L25

Cheng, X., Zhang, J., Saar, S. H., \& Ding, M. D. 2012a, ApJ, 761, 62

Cheng, X., Zhang, J., Olmedo, O., et al. 2012b, ApJ, 745, L5

Cheng, X., Ding, M. D., \& Fang, C. 2015, ApJ, 804, 82

Chifor, C., Tripathi, D., Mason, H. E., \& Dennis, B. R. 2007, A\&A, 472, 967

Cho, K., Lee, J., Chae, J., et al. 2016, Sol. Phys., 291, 2391

Dolla, L. R., \& Zhukov, A. N. 2011, ApJ, 730, 113

Dudík, J., Polito, V., Janvier, M., et al. 2016, ApJ, 823, 41

Fletcher, L., Dennis, B. R., Hudson, H. S., et al. 2011, Space Sci. Rev., 159, 19

Forbes, T. G., Linker, J. A., Chen, J., et al. 2006, Space Sci. Rev., 123, 251

Harra, L. K., \& Sterling, A. C. 2001, ApJ, 561, L215

Harra, L. K., Hara, H., Imada, S., et al. 2007, PASJ, 59, 801

Harrison, R. A., \& Lyons, M. 2000, A\&A, 358, 1097

Hudson, H. S. 2011, Space Sci. Rev., 158, 5

Imada, S., Hara, H., Watanabe, T., et al. 2007, PASJ, 59, 793

Imada, S., Hara, H., Watanabe, T., et al. 2011, ApJ, 743, 57

Jin, M., Ding, M. D., Chen, P. F., Fang, C., \& Imada, S. 2009, ApJ, 702, 27

Joshi, B., Veronig, A. M., Lee, J., et al. 2011, ApJ, 743, 195

Kahler, S. W., \& Hudson, H. S. 2001, J. Geophys. Res., 106, 29239

Lantos, P., Kerdraon, A., Rapley, G. G., \& Bentley, R. D. 1981, A\&A, 101, 33

Lemen, J. R., Title, A. M., Akin, D. J., et al. 2012, Sol. Phys., 275, 17

Li, T., \& Zhang, J. 2015, ApJ, 804, L8

Li, D., Ning, Z. J., \& Zhang, Q. M. 2015a, ApJ, 807, 72

Li, D., Ning, Z. J., \& Zhang, Q. M. 2015b, ApJ, 813, 59

Liu, W., \& Ofman, L. 2014, Sol. Phys., 289, 3233

Mason, J. P., Woods, T. N., Caspi, A., Thompson, B. J., \& Hock, R. A. 2014 ApJ, 789, 61

Miklenic, C., Veronig, A. M., Temmer, M., Möstl, C., \& Biernat, H. K. 2011, Sol. Phys., 273, 125

Moore, R. L., Sterling, A. C., Hudson, H. S., \& Lemen, J. R. 2001, ApJ, 552, 833

Patsourakos, S., \& Vourlidas, A. 2012, Sol. Phys., 281, 187

Patsourakos, S., Vourlidas, A., Wang, Y. M., Stenborg, G., \& Thernisien, A. 2009, Sol. Phys., 259, 49

Reinard, A. A., \& Biesecker, D. A. 2008, ApJ, 674, 576

Robbrecht, E., \& Wang, Y.-M. 2010, ApJ, 720, L88

Scherrer, P. H., Schou, J., Bush, R. I., et al. 2012, Sol. Phys., 275, 207

Sterling, A. C., \& Hudson, H. S. 1997, ApJ, 491, L55

Sterling, A. C., \& Moore, R. L. 2005, ApJ, 630, 1148

Sterling, A. C., Moore, R. L., \& Freeland, S. L. 2011, ApJ, 731, L3

Su, Y., Surges, V., van Ballegooijen, A., DeLuca, E., \& Golub, L. 2011, ApJ, 734,53

Su, Y., van Ballegooijen, A., McCauley, P., et al. 2015, ApJ, 807, 144

Thompson, B. J., \& Myers, D. C. 2009, ApJS, 183, 225

Thompson, B. J., Plunkett, S. P., Gurman, J. B., et al. 1998, Geophys. Res. Lett., 25,2465

Thompson, B. J., Cliver, E. W., Nitta, N., Delannée, C., \& Delaboudinière, J.-P. 2000, Geophys. Res. Lett., 27, 1431

Titov, V. S., \& Démoulin, P. 1999, A\&A, 351, 707

Veronig, A. M., Muhr, N., Kienreich, I. W., Temmer, M., \& Vršnak, B. 2010, ApJ, 716, L57

Warmuth, A. 2015, Li. Rev. Sol. Phys., 12, 3

Xia, C., Keppens, R., Antolin, P., \& Porth, O. 2014, ApJ, 792, L38

Zarro, D. M., Sterling, A. C., Thompson, B. J., Hudson, H. S., \& Nitta, N. 1999, ApJ, 520, L139

Zhang, J., Dere, K. P., Howard, R. A., Kundu, M. R., \& White, S. M. 2001, ApJ, 559,452

Zhang, J., Cheng, X., \& Ding, M.-D. 2012, Nat. Comm., 3, 747

Zhang, Q. M., Chen, P. F., Xia, C., \& Keppens, R. 2012, A\&A, 542, A52

Zhang, Q. M., Ning, Z. J., Guo, Y., et al. 2015, ApJ, 805, 4

Zhang, Q. M., Li, D., Ning, Z. J., et al. 2016, ApJ, 827, 27

Zhao, J., Gilchrist, S. A., Aulanier, G., et al. 2016, ApJ, 823, 62

Zhukov, A. N., \& Auchère, F. 2004, A\&A, 427, 705 